The Astrophysical Journal, 341 :L37-L40, 1989 June 15

(C) 1989. The American Astronomical Society. All rights reserved. Printed in U.S.A.

\title{
A NEW FAMILY OF DISTANCE INDICATOR RELATIONS FOR ELLIPTICAL GALAXIES
}

\author{
R. R. DE CARVALHO ${ }^{1}$ AND S. DJORgOVSKI ${ }^{2}$ \\ Division of Physics, Mathematics, and Astronomy, California Institute of Technology \\ Received 1989 March 14; accepted 1989 April 3
}

\begin{abstract}
We describe a new family of distance indicator relations for elliptical galaxies. Distance-dependent quantities, i.e., radius or luminosity, are expressed as distance-independent combinations of the mean surface brightness and a color, or any other metallicity indicator. Our preliminary results, based on a somewhat heterogeneous collection of published data, indicate that the typical relative distance errors to individual galaxies derived from new relations are $\sim 25 \%$, and the accuracy of $\sim 15 \%$ may be achievable with more homogeneous data sets. This is comparable to the accuracy obtained from the Tully-Fisher and $D_{n}-\sigma$ relations. The importance of the new relations is that the necessary data are derived from photometry only and thus can be obtained relatively quickly for large numbers of galaxies. This can lead to a more efficient and detailed mapping of the large-scale velocity field and streaming motions. A preliminary application of the new relations gives for the relative distance modulus between the Virgo and Coma clusters $\Delta(m-M)=3.95 \pm 0.25$, in a good agreement with measurements obtained using other methods. The new relations are interpreted in terms of the "fundamental plane" of elliptical galaxies, and imply the existence of a second parameter in the massmetallicity relation, identified here as the internal luminosity density.
\end{abstract}

Subject headings: galaxies: distances - galaxies: general

\section{INTRODUCTION}

Measurements of large-scale peculiar velocity fields are of a considerable importance in constraining the models of formation and evolution of galaxies and large-scale structure. In addition to redshifts, relative galaxy distances are required, and distance indicator relations such as the Tully-Fisher (1977, hereafter TF) are used to provide them.

The properties of elliptical galaxies form a two-parameter family, which can be represented by an inclined plane in the space defined by radius, velocity dispersion, and mean surface brightness (Dressler et al. 1987, hereafter D7S; Djorgovski and Davis 1987, hereafter DD87; Djorgovski 1987). The physical explanation behind this "fundamental plane" of early-type galaxies is simply that (a) galaxies are bound by Newtonian gravity and $(b)$ they have a similar dynamical structure (Djorgovski, de Carvalho, and Han 1988). The relation is very tight, and the relative error of predicted radii is typically $\sim 20 \%$ per galaxy. This makes the fundamental plane a better distance indicator relation in comparison with the FaberJackson (1976) relation, and possibly as good as the best versions of the TF relation for spirals.

Lynden-Bell et al. (1988, hereafter L7S) applied the fundamental plane (in its $D_{n}-\sigma$ form, proposed by D7S) to map the local velocity field. They found large peculiar motions relative to the microwave background on scales of tens of $\mathrm{Mpc}$, and a coherent infall in the direction of the Hydra-Centaurus supercluster (the "great attractor" model). If confirmed, their result would have important implications for theories of the formation and evolution of galaxies and large-scale structure. The amplitude of the motions claimed by L7S is probably incompatible with the cold dark matter scenario (Vittorio, Juskiewicz, and Davis 1986). Using the infrared TF relation, Aaronson et al. (1989) also find large peculiar motions in the

\footnotetext{
${ }^{1}$ On leave from Observatorio Nacional, $\mathrm{CNPq}$, Rio de Janeiro, Brasil

${ }^{2}$ Alfred P. Sloan Foundation fellow.
}

direction of Hydra-Centaurus, but there are important differences in their peculiar velocities of individual clusters, compared to the measurements by L7S. There is no consensus at this time on the exact nature of the large-scale peculiar velocity field on the scales of $\sim 100 \mathrm{Mpc}$ around us. An independent set of measurements for a large number of galaxies would be very valuable in clarifying the situation.

Motivated by the observation that colors of early-type galaxies correlate well with velocity dispersions (Tonry and Davis 1981; Wirth and Gallagher 1984), we attempted to substitute colors in place of velocity dispersions in the solutions for the fundamental plane in its $R-\sigma-\mu$ form (cf. DD87). Thus, we discovered a new family of distance indicator relations for elliptical galaxies, in which radius or luminosity is correlated with a linear combination of the mean surface brightness, and a metallicity term, e.g., a metallicity-sensitive color or the $\mathrm{Mg}_{2}$ index (de Carvalho and Djorgovski 1988, 1989a). A hint of the similar relation was already present in the work of Michard (1979), who did not pursue it further.

\section{THE DATA SETS AND ANALYSIS}

We use the effective radii $\left(r_{e}\right)$ and mean surface brightness $\left(\left\langle\mu_{e}\right\rangle\right)$ from the surface photometry by Djorgovski (1985a; see also DD87). The surface brightness is the average within the $r_{e}$ elliptical isophote, in the Lick red $r_{G}$ band (Djorgovski 1985b). We also use the $(U-B)$ and $(B-V)$ colors from de Vaucouleurs and Olson (1982, hereafter dVO), the $(u-V)$ and $(b-V)$ colors from Sandage and Visvanathan (1978, hereafter SV), and the $(V-K)$ colors from Persson, Frogel, and Aaronson (1979, hereafter PFA). In addition, we use the data set from Burstein et al. (1987, hereafter B7S), which contains the effective radii and mean surface brightness (in the $B$ band), and the $(B-V)$ colors, derived from photoelectric aperture photometry, as well as the spectroscopic measurements of the $\mathrm{Mg}_{2}$ index from D7S, which is a more direct estimator of the metallicity than are the broad-band colors. (Note that the effective radii have different operational definitions in DD87 and B7S.) 
We restrict the samples to the galaxies with low values of Galactic extinction $\left(A_{B}<0.5 \mathrm{mag}\right)$, determined from the maps by Burstein and Heiles (1982). Finally, we use the $(U-R)$ colors and the photographic $R$ band surface photometry of cluster galaxies by Strom and Strom $(1978 a, b, c$, hereafter collectively SS).

First, apparent radii ( $r_{e}$, in arcsec) are converted to absolute radii $\left(R_{e}\right.$, in pc), and the apparent magnitudes (within $\left.r_{e}\right)$ to the absolute magnitudes $\left(M_{e}\right)$, using the galaxy distances from the "great attractor" model by L7S. We assume $H_{0}=100 \mathrm{~km} \mathrm{~s}^{-1}$ $\mathrm{Mpc}^{-1}$ throughout. The choice of distances or the velocity field model is not critical for our present purpose, viz., a demonstration of the existence of the new relations. The question of peculiar velocities deduced from the application of these relations, will be addressed in a fucure paper. We search for the solutions of the form: $\log R_{e}=A$ (color) $+B\langle\mu\rangle+C$, and similar expressions for $M_{e}$. The fits were obtained by minimizing the dispersion in $\log R_{e}$. All points were given equal weight, since in most cases, the measurement errors are rather uncertain, or unavailable. The uncertainties in the slopes $A$ and $B$, estimated from the variations of $\chi^{2} / \chi_{\text {best fit }}^{2}$, are of the order of $10 \%$. The fits involving $M_{e}$ generally show a slightly larger scatter than the fits involving $R_{e}$.

A sample of solutions is listed in Table 1, including the relative scatter in predicted distances for each solution, expressed as $\Delta=\mathrm{rms}$ of $\ln r_{e}$. In the spite of a rather heterogeneous data base, there is a generally good agreement in the coefficients of compatible quantities - the solutions are fairly robust. This is reassuring, and a more homogeneous data base should produce even better fits. The scatter of predicted distances per galaxy is fairly low, generally in the range of $20 \%$ $30 \%$; this also includes the measurement errors, the peculiar velocities of individual galaxies, and any possible inadequacies of the model used to derive the absolute radii and magnitudes.

\section{DISCUSSION AND CONCLUSIONS}

The new radius-metallicity indicator-surface brightness relations can be understood as a generalization of the fundamental plane of elliptical galaxies: The velocity dispersion term there basically measures the depth of the galaxy's potential well. Since the metallicity of an elliptical galaxy also depends on the depth of the potential well, one can substitute a metallicity indicator in place of the velocity dispersion. The metallicity should be less affected by the internal dynamics (anisotropy, rotation) than the projected velocity dispersion. Some projections of this new version of the fundamental plane are illustrated in Figure 1. The importance of the new distance indicator relations is in the fact that colors are much easier to measure and calibrate than the velocity dispersions, and thus large numbers of galaxies can be surveyed relatively quickly.

The new relations work almost as well as the $D_{n}-\sigma$ relation of D7S and L7S. The typical scatter (relative errors of predicted distances) is about $25 \%-30 \%$ per galaxy, if the colors are used, as in the examples above. The scatter reduces to $\sim 15 \%-$ $20 \%$ if the $\mathrm{Mg}_{2}$ index from D7S is used, demonstrating that the metallicity is indeed the driving factor. However, the $\mathrm{Mg}_{2}$ index requires spectroscopy and is thus more expensive to measure and calibrate in terms of observing time. The scatter appears to be limited by the accuracy and homogeneity of the available data. From the same photometry, the scatter of the $D_{n}-\sigma$ relation as quoted by D7S and L7S is $22 \%-25 \%$. Our present results are based simply on the data available in the literature, and the $(B-V)$ color is not an ideal choice. One can improve the results by choosing a more metallicity-sensitive color, e.g., $(V-K)$, or the Gunn $(v-r)$.

Several effects can in principle contribute to the scatter in these relations. The assignment of distances (especially for the field galaxies) or the cluster membership can be a real problem in some cases. There may be age effects, caused, e.g., by recent star formation in some galaxies (Véron and Véron-Cetty 1985), or nonthermal sources of light, e.g., faint active nuclei. No attempt was made to preselect galaxies to guard against these effects in this preliminary report. We did check whether the presence of dust in these elliptical galaxies affects our fits, using the results of Ebneter, Djorgovski, and Davis (1988), and found no effect.

As an example, we use the data from B7S and D7S to derive the differential distance modulus between the Virgo and Coma clusters, as illustrated in Figure 2. Using the mean relation, involving the $(B-V)$ colors, we obtain $\Delta(m-M)=3.70$, and using the mean relation involving the $\mathrm{Mg}_{2}$ index, we obtain $\Delta(m-M)=4.15$. We adopt the average of the two solutions, $\Delta(m-M)=3.95 \pm 0.25$. These numbers are preliminary but are in good agreement with other determinations found in the literature: Dressler (1984) finds $\Delta(m-M)=3.75$ using the Faber-Jackson relation, and 4.00 using the luminosity $-\mathrm{Mg}_{2}$ index relation; Aaronson et al. (1986) find $3.69 \pm 0.16$ using the infrared TF relation; D7S find 3.65 using the $\bar{D}_{n}-\sigma$ relation;

TABLE 1

EXAMPles of New Distance INDicator Relations

\begin{tabular}{|c|c|c|c|}
\hline Sample & $N_{\text {gal }}$ & Relation & $\Delta$ \\
\hline \multirow[t]{2}{*}{ All $\ldots \ldots \ldots \ldots \ldots \ldots \ldots$} & 51 & $\log R_{e, \mathrm{DD}}=2.56(B-V)_{\mathrm{dvo}}+0.35\langle\mu\rangle_{e, \mathrm{DD}}-5.47$ & 0.29 \\
\hline & 57 & $\log R_{e, \mathrm{DD}}=2.16(b-V)_{\mathrm{SV}}+0.22\langle\mu\rangle_{e, \mathrm{DD}}-2.07$ & 0.35 \\
\hline Major Clusters & 57 & $\log R_{e, \mathrm{~B} 7 \mathrm{~S}}=3.12(B-V)_{\mathrm{B} 7 \mathrm{~S}}+0.38\langle\mu\rangle_{e, \mathrm{~B} 7 \mathrm{~S}}-7.15$ & 0.33 \\
\hline Virgo .................. & 17 & $\log r_{e, \mathrm{~B} 7 \mathrm{~S}}=3.25(B-V)_{\mathrm{B} 7 \mathrm{~S}}+0.45\langle\mu\rangle_{e, \mathrm{~B} 7 \mathrm{~S}}-10.5$ & 0.36 \\
\hline \multirow[t]{2}{*}{ Coma $\ldots \ldots \ldots \ldots \ldots$} & 31 & $\log r_{e, \mathrm{~B} 7 \mathrm{~S}}=3.30(B-V)_{\mathrm{B} 7 \mathrm{~S}}+0.37\langle\mu\rangle_{e, \mathrm{~B} 7 \mathrm{~S}}-9.78$ & 0.26 \\
\hline & 22 & $\log r_{e, \mathrm{~B} 7 \mathrm{~S}}=4.88 \mathrm{Mg}_{2, \mathrm{D} 7 \mathrm{~S}}+0.39\langle\mu\rangle_{e, \mathrm{~B} 7 \mathrm{~S}}-8.42$ & 0.20 \\
\hline \multirow[t]{2}{*}{ Virgo $\ldots \ldots \ldots \ldots \ldots \ldots$} & 16 & $\log r_{e, \mathrm{~B} 7 \mathrm{~S}}=5.78 \mathrm{Mg}_{2, \mathrm{D} 7 \mathrm{~S}}+0.28\langle\mu\rangle_{e, \mathrm{~B} 7 \mathrm{~S}}-5.80$ & 0.15 \\
\hline & 10 & $\log r_{e, \mathrm{DD}}=5.36 \mathrm{Mg}_{2, \mathrm{D} 7 \mathrm{~S}}+0.27\langle\mu\rangle_{e, \mathrm{DD}}-5.12$ & 0.14 \\
\hline Coma & 22 & $\log r_{e, \mathrm{ss}}=0.88(U-R)_{\mathrm{ss}}+0.19 \mu_{e, \mathrm{ss}}-5.27$ & 0.28 \\
\hline & 45 & $\log R_{e, \mathrm{~B} 7 \mathrm{~S}}=1.43(V-K)_{\mathrm{PFA}}+0.33\langle\mu\rangle_{e, \mathrm{~B} 7 \mathrm{~S}}-7.89$ & 0.27 \\
\hline \multirow[t]{5}{*}{ Virgo $\ldots \ldots \ldots \ldots \ldots$} & 14 & $\log r_{e, \mathrm{~B} 7 \mathrm{~S}}=2.02(V-K)_{\mathrm{PFA}}+0.34\langle\mu\rangle_{e, \mathrm{~B} 7 \mathrm{~S}}-12.2$ & 0.22 \\
\hline & 10 & $\log r_{e, \mathrm{DD}}=1.30(V-K)_{\mathrm{PFA}} 0.19\langle\mu\rangle_{e, \mathrm{DD}}-6.23$ & 0.21 \\
\hline & 17 & $M_{e, \mathrm{~B} 7 \mathrm{~S}}=13.3(B-V)_{\mathrm{B} 7 \mathrm{~S}}+1.20\langle\mu\rangle_{e, \mathrm{~B} 7 \mathrm{~S}}-16.4$ & 0.36 \\
\hline & 14 & $M_{e, \mathrm{~B} 7 \mathrm{~S}}=31.6 \mathrm{Mg}_{2, \mathrm{D} 7 \mathrm{~S}}+0.97\langle\mu\rangle_{e, \mathrm{~B} 7 \mathrm{~S}}-3.85$ & 0.34 \\
\hline & 14 & $M_{e, \mathrm{~B} 7 \mathrm{~S}}=8.97(V-K)_{\mathrm{PFA}}+1.20\langle\mu\rangle_{e, \mathrm{~B} 7 \mathrm{~S}}-18.6$ & 0.35 \\
\hline
\end{tabular}



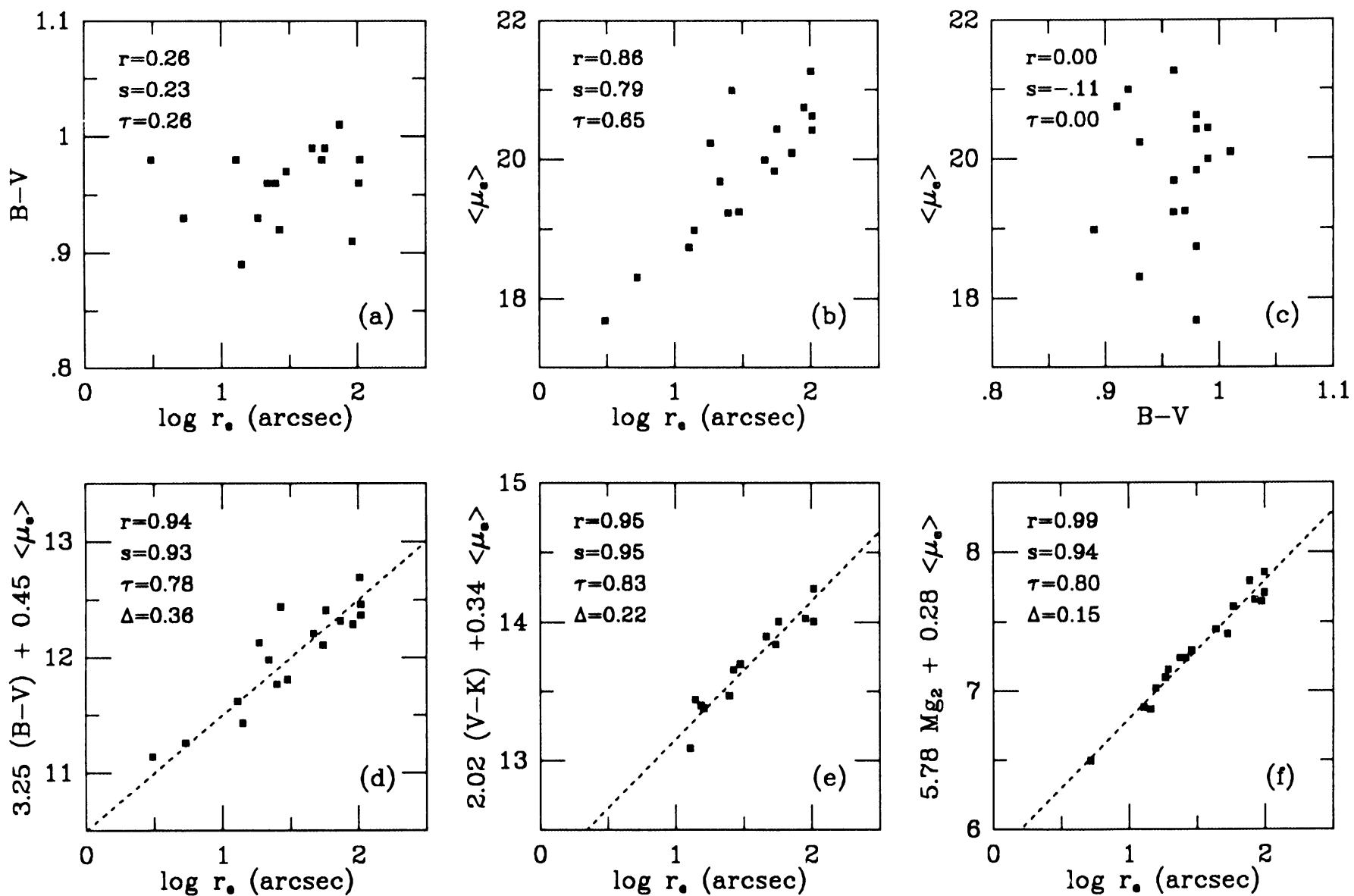

Fig. 1.-Projections of the radius-color-surface brightness plane, and examples of the new distance indicator relations, for a set of the Virgo cluster ellipticals The data are from the references indicated in the text: $(a)$ Projection on the $(B-V)$ color-radius plane; there is only a weak correlation. (b) Projection on the surface brightness-radius plane. This is a well-known correlation, but with a residual scatter (exceeding the measurement errors), which is correlated with the velocity dispersion or the color or metallicity. (c) Projection on the $(B-V)$ color-surface brightness plane; there is no discernible correlation. This is the color-defined version of the "fundamental plane" seen almost face-on. $(d)$ The new $(B-V)$ color-surface brightness-radius relation. This is the color-defined version of the "fundamental plane" seen edge-on. (e) Another version, using the $(V-K)$ color which is a better indicator of metallicity. $(f)$ Another version, using the $\mathrm{Mg}_{2}$ index as a metallicity indicator. Note the improvement in the scatter. In each panel, we list the correlation coefficients, linear regression $(r)$, Spearman rank (s), and Kendall rank $(\tau)$, and the rms scatter of $\ln R_{e},(\Delta)$, which is approximately equal to the relative fractional error in predicted distances.
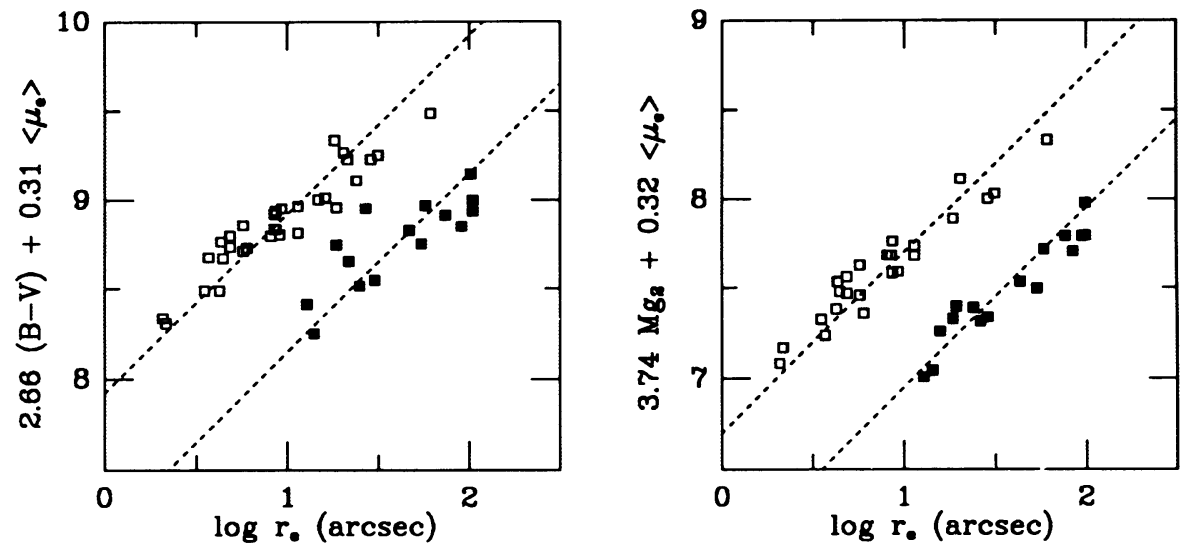

Fig. 2. - Comparison of the new distance indicator relations in the Virgo (solid squares) and Coma (open squares) clusters. The data are from D7S. (a) Fits to the relation $\log r_{e}=2.66(B-V)+0.31\left\langle\mu_{B}\right\rangle+$ const. The relative distance modulus derived is $\Delta(m-M)=3.70$. (b) Fits to the relation $\log r_{e}=3.74 \mathrm{Mg}_{2}+0.32$ $\left\langle\mu_{B}\right\rangle+$ const. The relative distance modulus derived is $\Delta(m-M)=4.15$. 
Kraan-Korteweg, Cameron, and Tammann (1988) find a range of values of 3.8-4.0 from their reanalysis of TF relation data; etc.

An important implication of the radius (or luminosity)surface brightness-metallicity relation is that there is a "second parameter" in the mass-metallicity relation for elliptical galaxies, which we identify as the internal luminosity density. Casting the new relations in that form may provide valuable constraints for theories of dissipative galaxy formation and initial enrichment (Djorgovski and de Carvalho 1989).

A possible remaining problem is the possibility that all of the known distance indicator relations, including the new ones presented here, may vary in different large-scale environments (e.g., field galaxies, groups, and rich clusters), or from one cluster to another. These relations between galaxian properties may well be modified by the processes of galaxy formation and transformation, many of which are environment-dependent: mergers, gas sweeping, tidal interactions, star formation history, etc. (cf. Djorgovski, de Carvalho, and Han 1988). Such hypothetical variations could masquerade as false peculiar velocities. Reassuringly, the coefficients of our new distance indicator relations evaluated separately in different clusters are the same, within the nominal errors. Still, a more detailed study is needed to explore this question.

It is a pleasure to thank J. Huchra, B. Madore, and M. Strauss for their constructive comments. We thank also D. Burstein for sending us the B7S data in a computer-readable form. R. R. dC. was supported by a CNPq fellowship, and California Institute of Technology. S. D. acknowledges a partial support from California Institute of Technology and from Alfred P. Sloan Foundation.
Aaronson, M., Bothun, G., Mould, J., Huchra, J., Schommer, R., and Cornell, M. 1986, Ap. J., 302, 536 .

Aaronson, M., et al. 1989, Ap. J., 338, 654.

Burstein, D., Davies, R., Dressler, A., Faber, S., Stone, R., Lynden-Bell, D. Terlevich, R., and Wegner, G. 1987, Ap. J. Suppl., 64, 601 (B7S). Burstein, D., and Heiles, C. 1982, A.J., 87, 1165.

de Carvalho, R., and Djorgovski, S. 1988, Bull. AAS, 20, 1037.

. 1989, in Workshop on Particle Astrophysics: Forefront Experimental

Issues, ed. E. Norman (Singapore: World Scientific), in press.

de Vaucouleurs, G., and Olson, D. 1982, Ap. J., 256, 346 (dVO).

Djorgovski, S. 1985a, Ph.D. thesis, University of California, Berkeley. 1985b, Pub. A.S.P., 97, 1119.

. 1987, in IAU Symposium 127, Structure and Dynamics of Elliptical Galaxies, ed. T. de Zeeuw (Dordrecht: Reidel), p. 79.

Djorgovski, S., and Davis, M. 1987, Ap. J., 313, 59 (DD87).

Djorgovski, S., and de Carvalho, R. 1989, in preparation.

Djorgovski, S., de Carvalho, R., and Han, M.-S. 1988, in Extragalactic Distance Scale, ed. S. van den Bergh and C. Pritchet (San Francisco: Astronomical Society of the Pacific), p. 329.

\section{REFERENCES}

Dressler, A. 1984, Ap. J., 281, 512.

Dressler, A., Lynden-Bell, D., Burstein, D., Davies, R., Faber, S., Terlevich, R., and Wegner, G. 1987, Ap. J., 313, 42 (D7S).

Ebneter, K., Djorgovski, S., and Davis, M. 1988, A.J., 95, 422.

Faber, S., and Jackson, R. 1976, Ap. J., 204, 668.

Kraan-Korteweg, R., Cameron, L., and Tammann, G. 1988, Ap. J., 331, 620.

Lynden-Bell, D., Faber, S., Burstein, D., Davies, R., Dressler, A., Terlevich, R., and Wegner, G. 1988, Ap. J., 326, 16 (L7S)

Michard, R. 1979, Astr. Ap., 74, 206.

Persson, E., Frogel, J., and Aaronson, M. 1979, Ap. J. Suppl., 39, 61 (PFA).

Sandage, A., and Visvanathan, N. 1978, Ap. J., 223, 707 (SV).

Strom, S., and Strom, K. 1978a, A.J., 83, 73 (SS).

$$
1978 b, \text { A.J., 83, } 732 \text { (SS). }
$$

. 1978c, A.J., 83, 1293 (SS)

Tonry, J., and Davis, M. 1981, Ap. J., 246, 680.

Tully, B., and Fisher, J. 1977, Astr. Ap., 54, 661 (TF).

Véron, P., and Véron-Cetty, M. 1985, Astr. Ap., 145, 433.

Vittorio, N., Juszkiewicz, R., and Davis, M. 1986, Nature, 323, 132.

Wirth, A., and Gallagher, J. 1984, Ap. J., 282, 85.

R. DE CARvalho: Robinson Laboratory, MS 105-24, California Institute of Technology, Pasadena, CA 91125 [SPAN: reinaldo@6035]

S. DJORGovsKi: Robinson Laboratory, MS 105-24, California Institute of Technology, Pasadena, CA 91125 [SPAN: george@6035] 